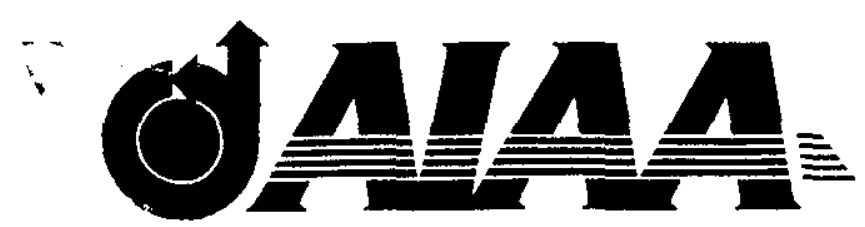 \\ AIAA 92-0284 \\ Prediction of Average Downwash Gradient for Canard Configurations \\ David W. Levy \\ The University of Michigan \\ Department of Aerospace Engineering Ann Arbor, MI
}

\section{0th Aerospace Sciences Meeting \& Exhibit January 6-9, 1992 / Reno, NV}




\title{
Prediction of Average Downwash Gradient for Canard Configurations
}

\author{
David W. Levy * \\ The University of Michigan \\ Department of Aerospace Engineering
}

\section{Abstract}

Design charts are presented which give correction factors to account for the nonlinear variation of downwash gradient induced by a canard across the span of the main wing. The correction factors are derived using the vortex lattice computational method to calculate the induced velocities behind the canard. Data are presented for a variety of relative positions and canard geometry. The method is valid for incompressible flow and for geometries where the canard wake does not impinge on the main wing. Comparisons with existing methods and results for canard/wing combinations indicate the method is accurate enough for conceptual and preliminary phases of the design of airplanes with canards.

\section{Nomenclature}

\begin{tabular}{ll}
$A R$ & Aspect ratio \\
$b$ & Span \\
$c$ & Chord \\
$C_{L}$ & Lift Coefficient \\
$C_{m}$ & Pitching Moment Coefficient \\
$k_{b}$ & Span Ratio Correction Factor \\
$S$ & Area \\
VLM & Vortex Lattice Method \\
$x$ & Longitudinal distance from quarter-chord \\
$y$ & Lateral distance from plane of symmetry \\
$z$ & Vertical distance from wing plane \\
$\alpha$ & Angle of attack \\
$\epsilon$ & Downwash angle \\
$\lambda$ & Taper ratio \\
$\Lambda$ & Sweep angle \\
Subscripts: \\
\hline$a c$ & Aerodynamic center \\
$c$ & Canard \\
$h$ & Horizontal tail \\
$r e f$ & Reference
\end{tabular}

*Lecturer; Senior Member, AIAA. Copyright (c) 1992 by the American Institute of Aeronautics and Astronautics, Inc. All rights reserved.

$\begin{array}{ll}w & \text { Wing } \\ w c & \text { Wing and canard }\end{array}$

\section{Introduction}

In the conceptual and preliminary phases of airplane design, aerodynamic forces and moments are often predicted using the "component build-up" method (see Roskam [1]). In this procedure, the total aerodynamic force or moment is approximated by the sum of contributions from the individual airplane components. For a conventional configuration with an aft tail, the lift curve slope, $C_{L_{\alpha}}$, of the wing-tail combination can be represented as the sum of the wing and tail contributions:

$$
C_{L_{\alpha}}=C_{L_{\alpha w}}+C_{L_{\alpha h}} \frac{S_{h}}{S_{w}}
$$

where it is seen that one must account for the tail planform area when adding the tail lift curve slope.

In general, however, the presence of one component will interfere with another. Then direct linear superposition will not give the correct result. In the case of the airplane lift coefficient, the wing will influence the tail because the wing generates a wake. One dominant effect of the wake is the downwash field, shown in Figure 1. The usual way to account for the downwash is to compute the average downwash at the location of the tail. Then equation 1 is modified to include the effect of the downwash field:

$$
C_{L_{\alpha}}=C_{L_{\alpha w}}+C_{L_{\alpha h}}\left(1-\frac{d \epsilon}{d \alpha}\right) \frac{S_{h}}{S_{w}}
$$

The average downwash gradient, $\frac{d \epsilon}{d \alpha}$, reduces the local angle of attack at the tail. The result is that the tail effectiveness is reduced, often by as much as $30 \%$ to $50 \%$. In principle, the horizontal tail will influence the wing as well. However, in practice this effect is very small and is neglected.

The prediction of the total pitching moment coefficient is similar, including the treatment of the downwash terms. The accurate prediction of the average 


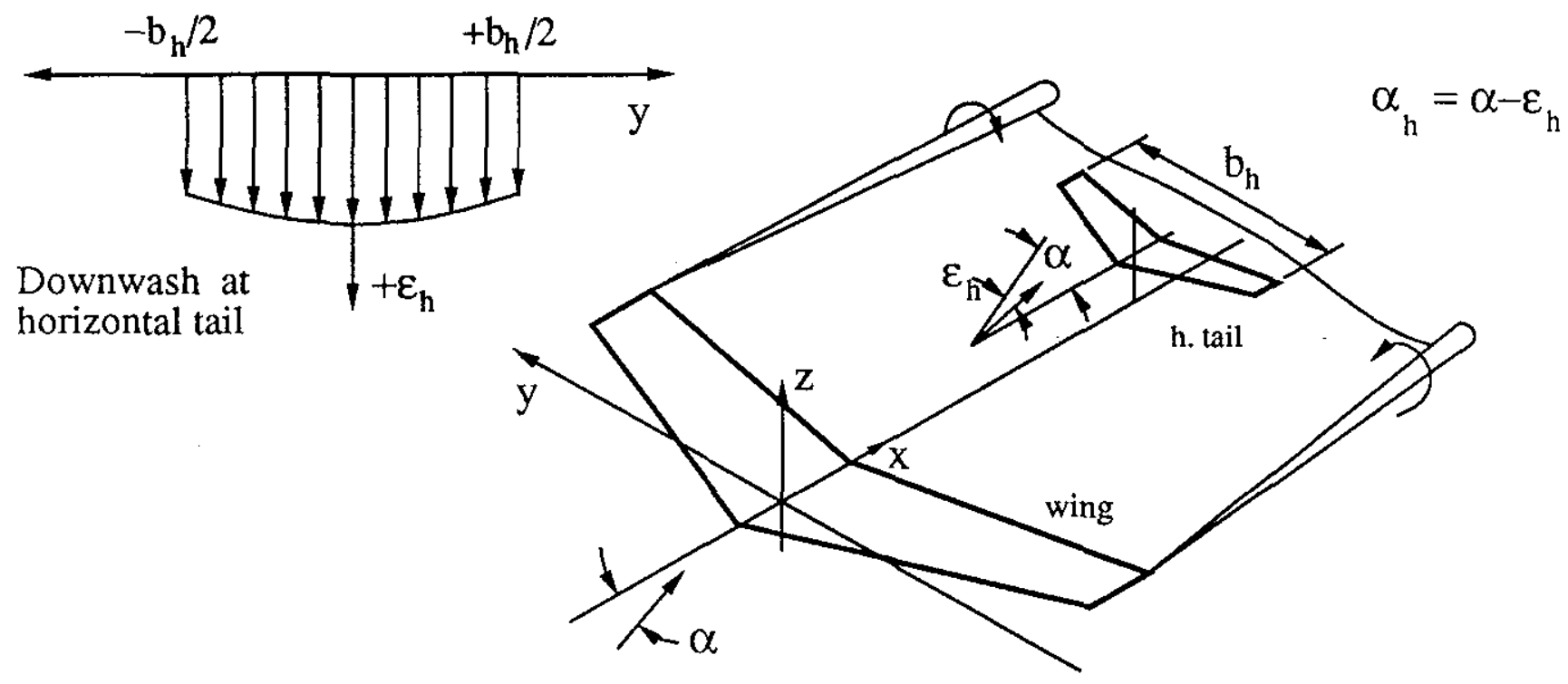

Figure 1: Downwash Field due to the Wing at the Horizontal Tail.

downwash gradient is very important in the determination of the longitudinal stability of a proposed airplane configuration.

The downwash field behind a wing, and methods for accounting for the effects on a wing in that field, are documented in several references. These include Roskam [1], Silverstein [2, 3], the DATCOM [4], and Phillips [5]. However, these references emphasize the case where an aft tail has a much smaller span than the main wing. The infuence of the wing on the tail is calculated by first estimating the downwash gradient in the plane of symmetry at the longitudinal and vertical location of the tail, and then correcting for the spanwise variation across the tail. The data in Roskam [1] and Silverstein [2] are limited to a span ratio, $b_{h} / b_{w}$, less than 0.4 . In this case the spanwise variation of the downwash gradient is relatively small (as shown in Figure 1).

In the case of a canard, where the forward wing has a smaller span than the aft wing, the spanwise variation of the downwash gradient at the aft wing is quite significant. This is shown in Figure 2. Most of the above references do not present methods to account for this nonlinear variation across the span. There is a rather cumbersome method in the DATCOM which is to be used for canard configurations, but it is primarily aimed at configurations which have low aspect ratios, and where body effects are significant. A simpler method is used by Phillips [6], where the reverse flow theorem from linearized compressible flow theory (see Ursell and Ward [7]) is applied. The reverse flow theorem states that for two lifting surfaces, such as a canard and a wing, the influence of the canard on the wing is the same as the influence of the wing on the canard with the flow reversed. This procedure transforms a canard configuration into a conventional tail-aft configuration. This method, however, would be difficult to use when the wing is swept aft, which is a fairly common aspect of pure canard configurations. Then the downwash field of a wing with forward sweep must be calculated, for which there is no available method.

It is desired to treat the effects of canard downwash in a manner similar to that for a horizontal tail. Then the component build-up technique will still be usable. The question then is: "What is the effective downwash gradient at the main wing?" The most straightforward approach is simply to use the average value.

This paper presents design charts for the determination of average downwash gradients for canard configurations including the effects of relative position, span ratio, area ratio, and aft wing sweep. Example applications are shown, and comparisons are made to computational results for wing-canard configurations. A primary goal is to present the data in a format that is easy to use and accurate enough for the conceptual and preliminary phases of airplane design.

\section{Method Description}

The Vortex Lattice Method, or VLM, (see Katz and Plotkin [8]) is used to solve for the flow field of each case under consideration. The use of distributions of horseshoe and ring vortices is widely accepted as a reasonably accurate model of the inviscid flow past a wing. It is fairly easy to use and the computations can be performed on modern workstation computers. 


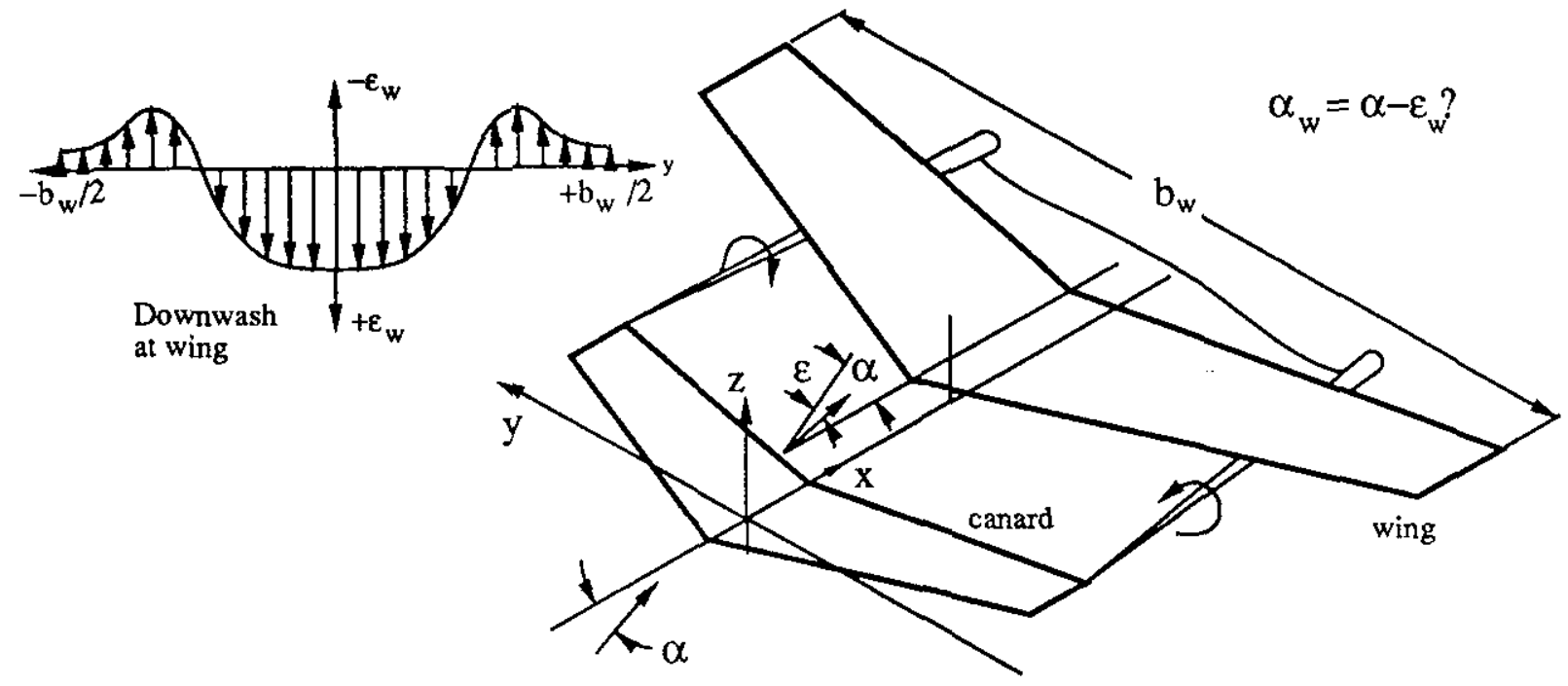

Figure 2: Downwash Field due to the Canard at the Wing.

In the particular implementation used here, each lifting surface is represented as a flat plate divided into many panels. A ring vortex is placed at each panel except those at the trailing edge. Here a horseshoe vortex is placed with the trailing filaments extending downstream to infinity. This is necessary to enforce the Kutta condition.

The leading edge of each vortex lies along the quarter-chord line of the corresponding panel. The strength of each vortex filament is determined by enforcing boundary conditions of zero normal velocity at the midpoint of the three-quarter chord line of each panel.

To improve the accuracy of the solution, the locations of the trailing filaments are found in an iterative fashion. Initially, the trailing filaments extend to infinity at an angle corresponding to the freestream angle of attack. With the solution found by this representation of the wake, the locations of the trailing filaments are relaxed, so that they follow the local streamlines. The solution is then recalculated with the new wake geometry. In practice, it is found that only one or two relaxation steps are necessary to obtain an accurate wake representation. A typical example of the grid and wake are shown in Figure 3.

With this software, the vorticity distribution may be calculated for a wing of arbitrary aspect ratio, taper ratio, and sweep angle. Once the vorticity distribution is known, the vortex induced velocity, and therefore the local downwash angle, can be calculated. Since the solution for a single case is completed in approximately ten minutes, it is possible to investigate a wide variety of planform geometries. With a systematic approach, trends in important variables can be identified. Once the principal effects have been identified, an ap- propriate format for presentation of the data can be constructed.

Due to the use of inviscid vortices to model the flow, certain limitations of the methodology warrant discussion. First, the velocity induced by a potential vortex is singular at points on the vortex. Therefore the velocity calculated at a point very near a vortex will be in error. Second, it is not possible to include the effects of a viscous wake. Third, the results include only canard-wing interactions. The presence of a fuselage is not accounted for. Some of these areas will be discussed further in later sections of this paper.

\section{Baseline Comparisons}

Before proceeding, it is worthwhile to check the performance of the VLM method as currently implemented. As a first check, the lift curve slope for an isolated, straight tapered wing can be computed. The empirical method recommended by the DATCOM [4] is the Polhamus formula. For incompressible flow and assuming a two-dimensional airfoil lift curve slope of $2 \pi$ per radian:

$$
C_{L_{\alpha}}=\frac{2 \pi A}{2+\sqrt{A^{2}\left(1+\tan ^{2} \Lambda_{c / 2}\right)+4}}, \operatorname{Rad}^{-1} .
$$

Values for a range of aspect ratio, taper ratio, and quarter chord sweep angle is shown in Table 1. The agreement is very good with an average magnitude difference of $2.8 \%$. There is a small dependence on the taper ratio in the VLM results, which is not predicted by the Polhamus formula.

Next, it is possible to compare the downwash gradient predicted at the plane of symmetry. This is accom- 


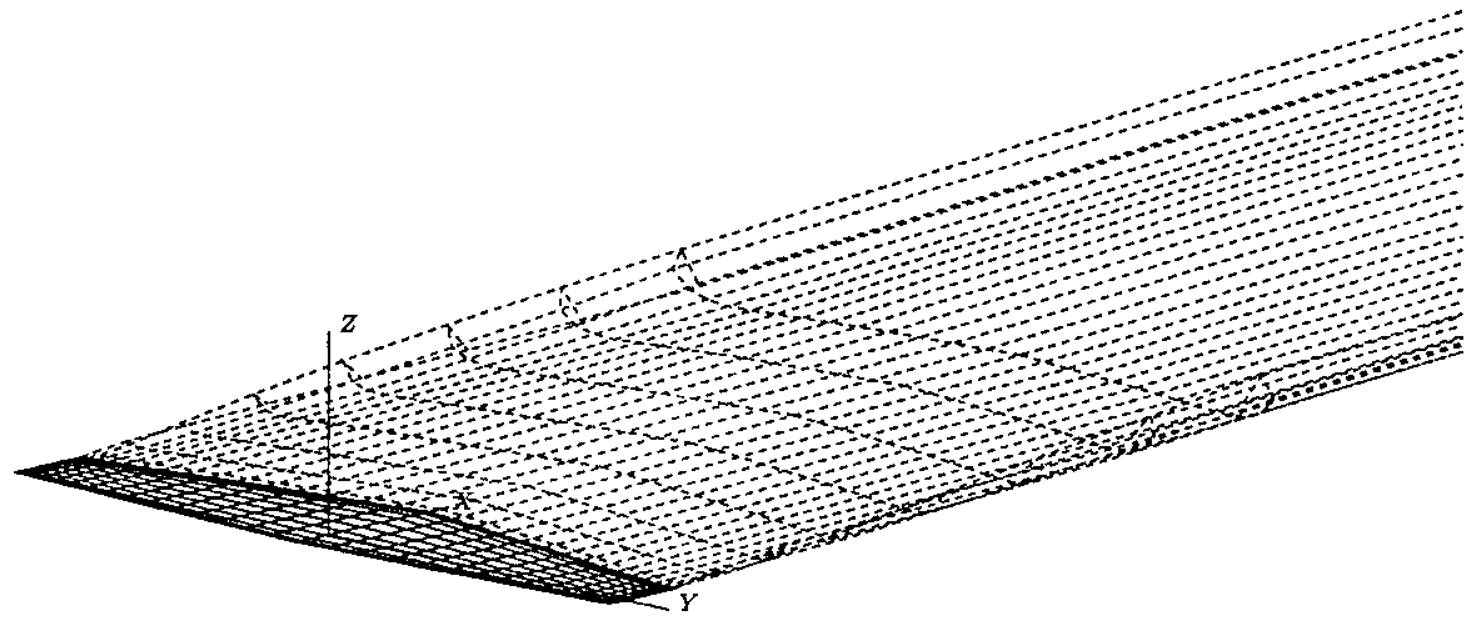

Figure 3: Example of $10 \times 15$ Grid for Wing With $A R=6, \lambda=0.50, \Lambda_{c / 4}=0^{\circ}$; After Two Wake Relaxation Steps.

\begin{tabular}{|c|c|c|c|c|c|}
\cline { 4 - 6 } \multicolumn{2}{c|}{} & \multicolumn{3}{c|}{ VLM for $\lambda=$} \\
\hline$A R$ & $\Lambda_{c / 2}$ & Empirical & 1.00 & .50 & .20 \\
\hline 6 & $0^{\circ}$ & .0790 & .0740 & .0764 & .0764 \\
\hline 9 & $0^{\circ}$ & .0880 & .0829 & .0854 & .0856 \\
\hline 12 & $0^{\circ}$ & .0929 & .0880 & .0905 & .0907 \\
\hline 6 & $30^{\circ}$ & .0714 & .0676 & .0696 & .0697 \\
\hline 9 & $30^{\circ}$ & .0784 & .0744 & .0766 & .0767 \\
\hline 12 & $30^{\circ}$ & .0822 & .0783 & .0804 & .0806 \\
\hline
\end{tabular}

Table 1: Lift Curve Slope (in $\mathrm{deg}^{-1}$ ) Comparison for Straight Tapered Wings.

plished by computing the downwash angles for an angle of attack of 5 degrees. Since there is no airfoil camber, the downwash gradient is simply the ratio of the downwash angle to the angle of attack. This assumes the gradient is linear for this angle of attack range. Calculations for an aspect ratio of 9 and a taper ratio of 0.2 is shown in Figure 4.

The data from Roskam [1] is derived from the experimental data in Silverstein and Katzoff [2], and the empirical method is from the DATCOM [4]. There are significant differences between all three methods for the cases shown. The DATCOM method predicts somewhat lower values for $d \epsilon / d \alpha$ than either the VLM results or the data from Roskam. Also, the change due to vertical position given by the DATCOM is much smaller.

The difference which is perhaps most significant is that the curves given by VLM for $z /(b / 2)=0.0$ and 0.1

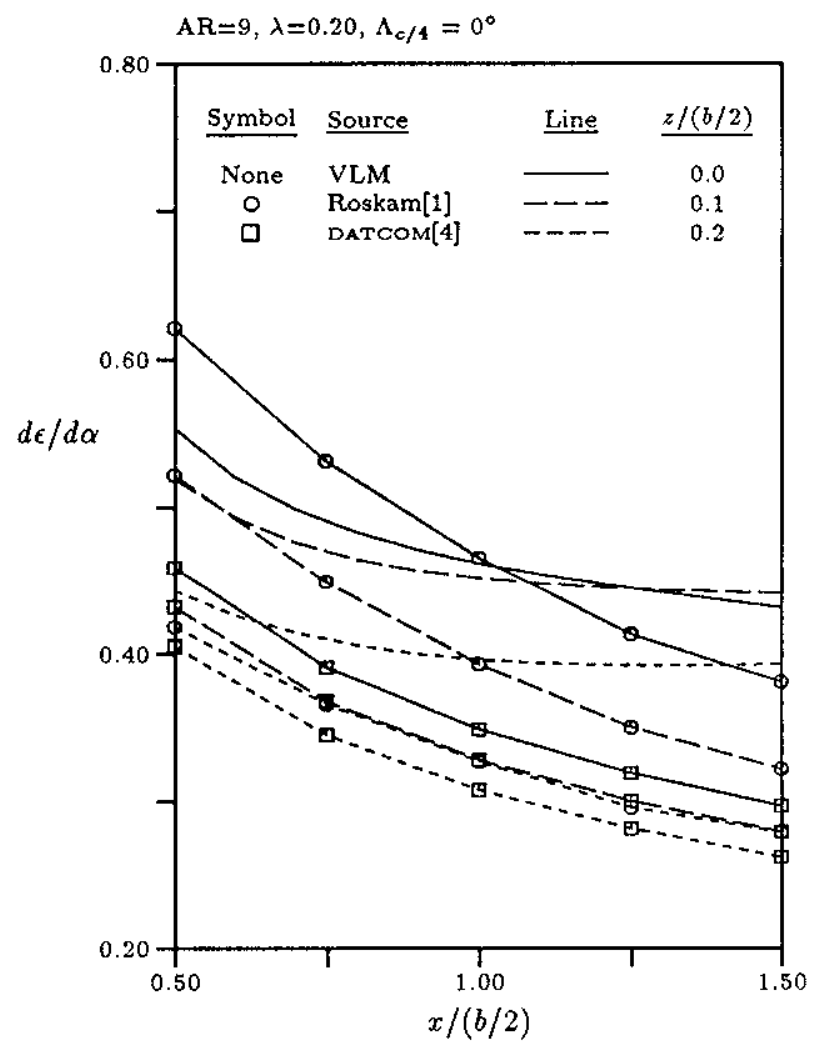

Figure 4: Comparison of Downwash Gradient at the Plane of Symmetry Given by the Vortex Lattice Method with Experimental and Empirical Data. 

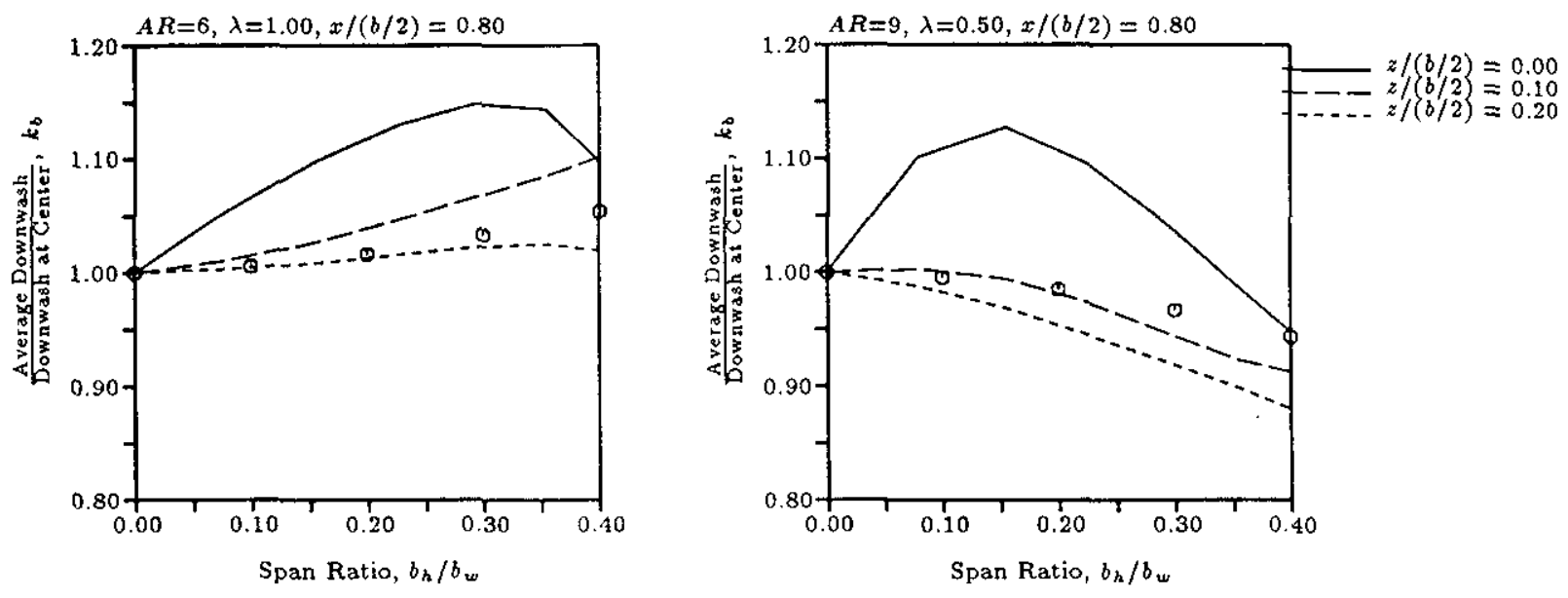

Symbols indicate data from Silverstein and Katzoff [2]

Figure 5: Correction to $d \epsilon / d \alpha$ in the Plane of Symmetry due to Variation Across the Span.

cross at $x /(b / 2)=1.25$. Neither of the other two methods predict this behavior. However, this phenomenon is easily explained when it is realized that the downwash gradient will be at a maximum at a point approximately on the vortex sheet. The sheet is curved, so the precise location of that maximum point is not easily defined. It can be stated that the maximum will be at a point between the chord plane and a plane aligned with the freestream angle of attack which intersects the trailing edge.

Since the vertical distance is defined relative to the chord plane, it is expected that the curves for $z /(b / 2)=$ 0.0 and 0.1 will cross at the longitudinal station where the maximum gradient is located at $z /(b / 2)=0.05$. It would seem that the experimental and empirical methods give data defined relative to the vortex sheet rather than the chord plane, but the references indicate that the vertical coordinate is defined relative to the chord plane.

As a third and final check, the factor to correct for spanwise variation in downwash gradient across the tail can be computed. The correction factor is simply the ratio of the average downwash gradient across the span of the tail to the value at the plane of symmetry. This is shown in Figure 5, along with data from Silverstein and Katzoff [2]. The reference data are derived in a manner similar to the present method.

The reference data are for a tail location corresponding to 0.75 semispan, while data for the present method is for 0.80 semispan, which is the closest available location. The vertical location of the reference data is "on the vortex sheet." It is seen that there is a strong dependence on vertical location.

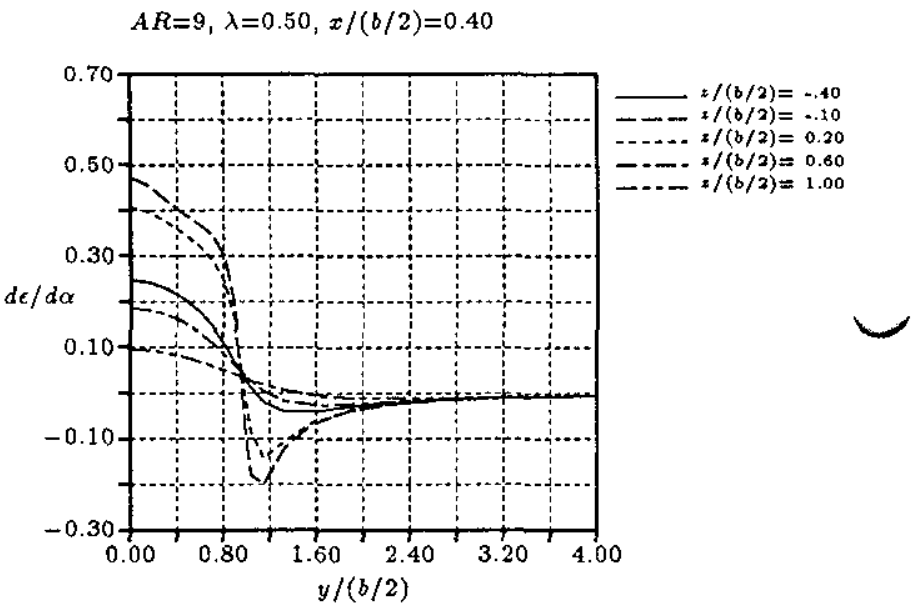

Figure 6: Variation in Local Downwash Gradient in Spanwise Direction.

\section{Presentation of Data}

An example of the variation of local downwash in the spanwise direction is shown in Figure 6. As expected, the magnitude of the downwash gradient decreases as the spanwise station progresses to the wingtip. Outboard of the wingtip, upwash is produced, and eventually the magnitude decreases back towards zero as the spanwise station becomes very large.

These data are only useful as a confirmation of the trends in downwash with spanwise location; they are not appropriate for use in the component build-up method. The format chosen here for the design charts is similar to that in Silverstein and Katzoff [2]. In general, the average downwash gradient is given as the value at 
the plane of symmetry multiplied by a correction factor which is dependent on the ratio of the wing span to the canard span:

$$
\frac{d \epsilon_{w}}{d \alpha}=\left.k_{b} \frac{d \epsilon_{w}}{d \alpha}\right|_{y=0}
$$

The downwash gradients at the plane of symmetry are presented as contour plots, where lines of constant $d \epsilon / d \alpha$ are shown in the $x-z$ plane. Results are presented for aspect ratio 6,9 , and 12 ; taper ratio of 1.0 , 0.50 , and 0.20 ; and quarter-chord sweep angles of 0 and 30 degrees in Figures 9-11.

The correction factor due to the variation in downwash gradient in the spanwise direction is presented next. The same ranges of aspect ratio, taper ratio and sweep angle are given. Results for these data ranges are given for longitudinal stations of $x /(b / 2)=.5,1.0$ and 2.0, in Figures 12-20. In a few cases for $x /(b / 2)$ $=2.0$ and $z /(b / 2)=0.2$, the correction curves exhibit behavior somewhat different from the others. In these cases the data are for locations too close to the vortex sheet. Here the data are less accurate because the vortex filaments use an inviscid model and the induced velocities are unrealistically large. To obtain data in these locations it is better to interpolate between data at other locations.

\section{Method of Application}

As a test case, the lift curve for a wing-canard configuration can be calculated. The test case chosen is for wing and canard aspect ratios and taper ratios equal to 6 and 0.50 , respectively, and for a wing location of 1.5 canard semispans behind and 0.6 canard semispans above the canard root quarter-chord position. These distances are measured in and normal to the canard root chord plane. For canards with cambered airfoil sections, the distances should be relative to the canard root zero lift plane.

The equation for the lift curve slope is similar to equation 2 :

$$
C_{L_{\alpha_{w c}}}=C_{L_{\alpha_{w}}}\left(1-\frac{d \epsilon_{w}}{d \alpha}\right)+C_{L_{\alpha_{c}}} \frac{S_{c}}{S_{w}}\left(1+\frac{d \epsilon_{c}}{d \alpha}\right) .
$$

It is seen that the influence upwash at the canard due to the wing is also included. Now, $\left(1-\frac{d \epsilon_{w}}{d \alpha}\right)$ is interpreted as the effective, or average value. The lift curve is constructed by integrating the the slope, assuming that $C_{L}$ at $\alpha=0$ is zero because both wing and canard have zero camber. For this case:

$$
\begin{aligned}
C_{L_{\alpha_{w}}} & =.0790 \mathrm{deg}^{-1} \\
C_{L_{\alpha_{c}}} & =.0790 \mathrm{deg}^{-1} \\
\frac{S_{c}}{S_{w}} & =0.25
\end{aligned}
$$

$$
\frac{d \epsilon_{c}}{d \alpha}=0.05 \text { (from [1]) . }
$$

The average downwash at the wing is the value at the plane of symmetry, $\left.\frac{d \epsilon_{y}}{d \alpha}\right|_{y=0}=0.27$ from Figure 9 , multiplied by the correction due to spanwise variation, $k_{b}=0.28$ interpolated from Figures 13 and 14:

$$
\frac{d \epsilon_{w}}{d \alpha}=0.28 \times 0.27=0.924 .
$$

Substitution into equation 5 gives:

$$
C_{L_{\alpha_{w c}}}=.0937 \mathrm{deg}^{-1} \text {. }
$$

Also of interest is the lift curve slope with no correction due to mutual interference (i.e. $\frac{d \epsilon_{w}}{d \alpha}=\frac{d \epsilon_{c}}{d \alpha}=0$ ), which is computed to be $.0988 \mathrm{deg}^{-1}$.

The pitching moment characteristics are usually presented in terms of the parameter $\frac{d C_{m}}{d C_{L}}$, where:

$$
\frac{d C_{m}}{d C_{L}}=\frac{C_{m_{\alpha}}}{C_{L_{\alpha}}}=\bar{x}_{r e f}-\bar{x}_{a c} .
$$

The " ' notation denotes distance as a fraction of the wing mean geometric chord. The location of the aerodynamic center with the influence of downwash at the wing due to the canard and upwash at the canard due to the wing can be determined in a manner similar to that in Roskam [1]. The formula for the aerodynamic center location is presented here without proof:

$$
\bar{x}_{a c_{w c}}=\frac{\bar{x}_{a c_{w}}+\frac{C_{L_{\alpha_{c}}}}{C_{L_{\alpha_{w}}}} \frac{S_{c}}{S_{w}} \frac{\left(1+\frac{d \epsilon_{c}}{d \alpha}\right)}{\left(1-\frac{d \epsilon_{w}}{d \alpha}\right)} \vec{x}_{a c_{c}}}{1+\frac{C_{L_{\alpha_{c}}}}{C_{L_{\alpha_{w}}}} \frac{S_{c}}{S_{w}} \frac{\left(1+\frac{d c_{c}}{d \alpha}\right)}{\left(1-\frac{d \epsilon_{w}}{d \alpha}\right)}} .
$$

All quantities have been calculated except for the aerodynamic center locations of the wing and canard. For this geometry, the nondimensional distances relative to the wing mean geometric chord leading edge are

$$
\begin{aligned}
& \bar{x}_{a c_{w}}=0.25 \\
& \bar{x}_{a c_{c}}=-1.919 .
\end{aligned}
$$

The lift and pitching moment lines are shown in Figure 7 compared with VLM results for the same configuration, and with the prediction without any correction for downwash. The agreement between the VLM results and the results with downwash included is very good. It is seen that the downwash reduces the lift by $5.4 \%$. The difference between the lift curve slopes predicted by the present method and the VLM is $5.9 \%$. The VLM predicts slightly lower values of lift, but recall from Table 1 that the lift for the isolated wing is also low. If the slope predicted by the Polhamus formula is lowered to correspond with the slope predicted by the VLM, then the difference drops to $2.5 \%$. This 

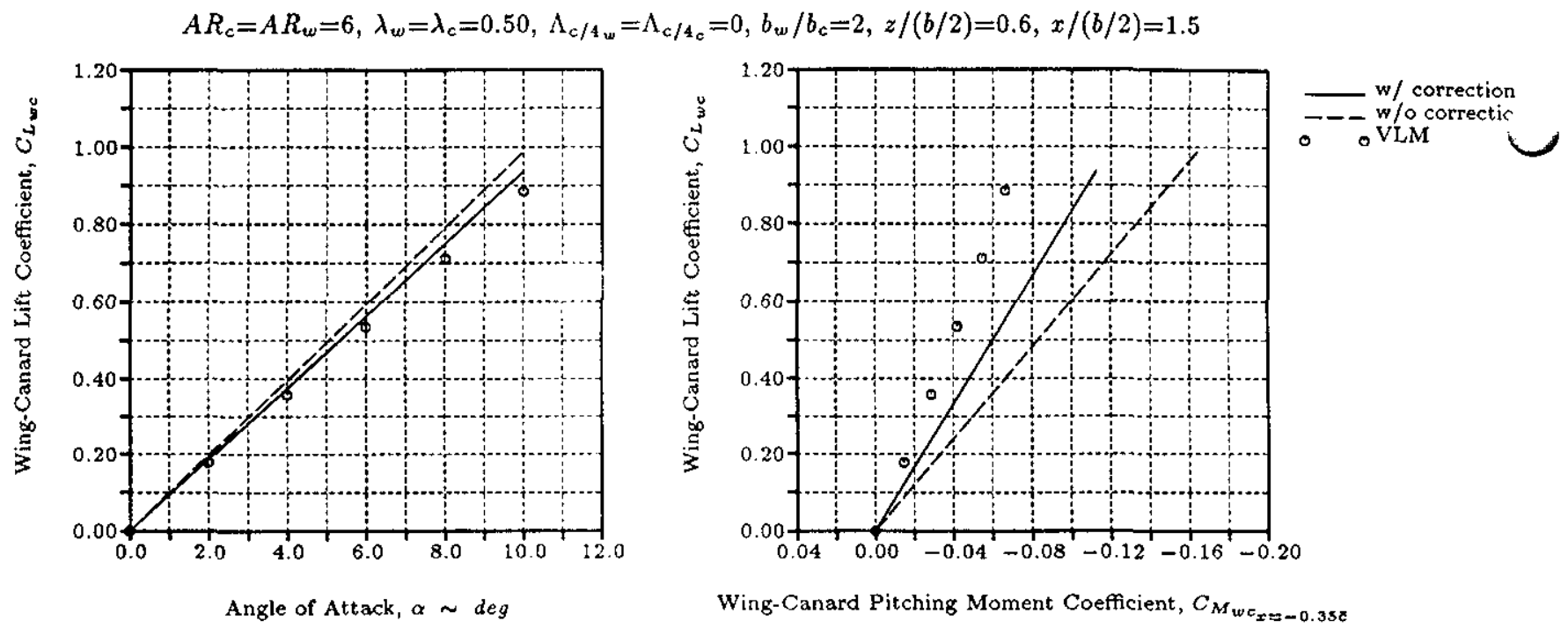

Wing-Canard Pitching Moment Coefficient, $C_{M} c_{x=-0.35 \varepsilon}$

Figure 7: Predicted Lift and Pitching Moment Curves for a Wing-Canard Configuration With and Without Downwash Correction, Compared with Vortex Lattice Method Results, $\Lambda_{c / 4_{w}}=0^{\circ}$.

\begin{tabular}{|c|c|c|}
\cline { 2 - 3 } \multicolumn{1}{c|}{} & \multicolumn{2}{c|}{$\bar{x}_{a c_{w c}}$} \\
\cline { 2 - 3 } \multicolumn{1}{c|}{} & $\Lambda_{c / 4_{w}}=0^{\circ}$ & $\Lambda_{c / 4_{w}}=30^{\circ}$ \\
\hline With Correction & -0.230 & -.239 \\
\hline Without Correction & -0.184 & -.192 \\
\hline VLM & -0.275 & -.263 \\
\hline
\end{tabular}

Table 2: Wing-Canard Aerodynamic Center Location, in $\% \bar{c}_{w}$, Relative to the Wing Mean Geometric Chord Leading Edge.

remaining error is attributed to the changes in spanwise lift distribution on the wing.

The pitching moment data shown are referenced to a point $0.35 \bar{c}_{w}$ ahead of the wing mean geometric chord leading edge. The data shown do not seem to agree well, however it must be realized that the relative error is dependent on the location of the reference point chosen. The static margin for a center of gravity located at this reference point is approximately $12 \%$, so a difference of just a few percent in the predicted aerodynamic center location will have a large relative effect. It is better to compare the aerodynamic center location directly, as shown in Table 2. The effect of the downwash and upwash correction is to move the aerodynamic center aft $4.6 \%$. The VLM still predicts a location $4.5 \%$ further aft than the corrected value.

While the lift curve is linear it is seen that the pitching moment curve predicted by the VLM exhibits slightly nonlinear behavior. As the angle of attack increases, the wake of the canard moves closer to the wing. In all cases, for this location of the wing relative to the canard, the wake passes safely below the wing. However, as the wake moves closer to the wing, its influence becomes stronger and a slightly less negative pitching moment results. The present method neglects the change in wake position with angle of attack. All the data shown in the Figures 9-20 are calculated for an angle of attack of five degrees.

As a second application, a case where the wing is swept back is considered. This is fairly common for canard-wing configurations with pusher engine installations. Sweeping the wing aft is then usually the best way to satisfy longitudinal stability requirements (i.e. move the airplane aerodynamic center aft). The geometry chosen is very similar to the first case except that the wing sweep angle at the quarter-chord is 15 degrees. In this case, it is recommended that the horizontal distance be measured to the aerodynamic center of the wing. It is recognized that if an average downwash is to be used, it would be proper to average it along the wing quarter-chord line. The spanwise correction data contained in this paper are averaged along lines parallel to the $y$ axis. If these data are used directly, then two effects are ignored: (1) the variation in downwash and upwash with longitudinal position and (2) the moment which is generated by the downwash on the inboard portion of the wing which is ahead of the wing a.c. coupled with the upwash on the outboard portion of the wing, which is aft of the a.c. It is assumed that the wing a.c. location is the proper position to use to compute an average net downwash.

The relative position of the wing and canard is set so that the vertical and horizontal distances to the wing a.c. are the same as in the previous example. All parameters in equations 5 and 9 remain unchanged with 

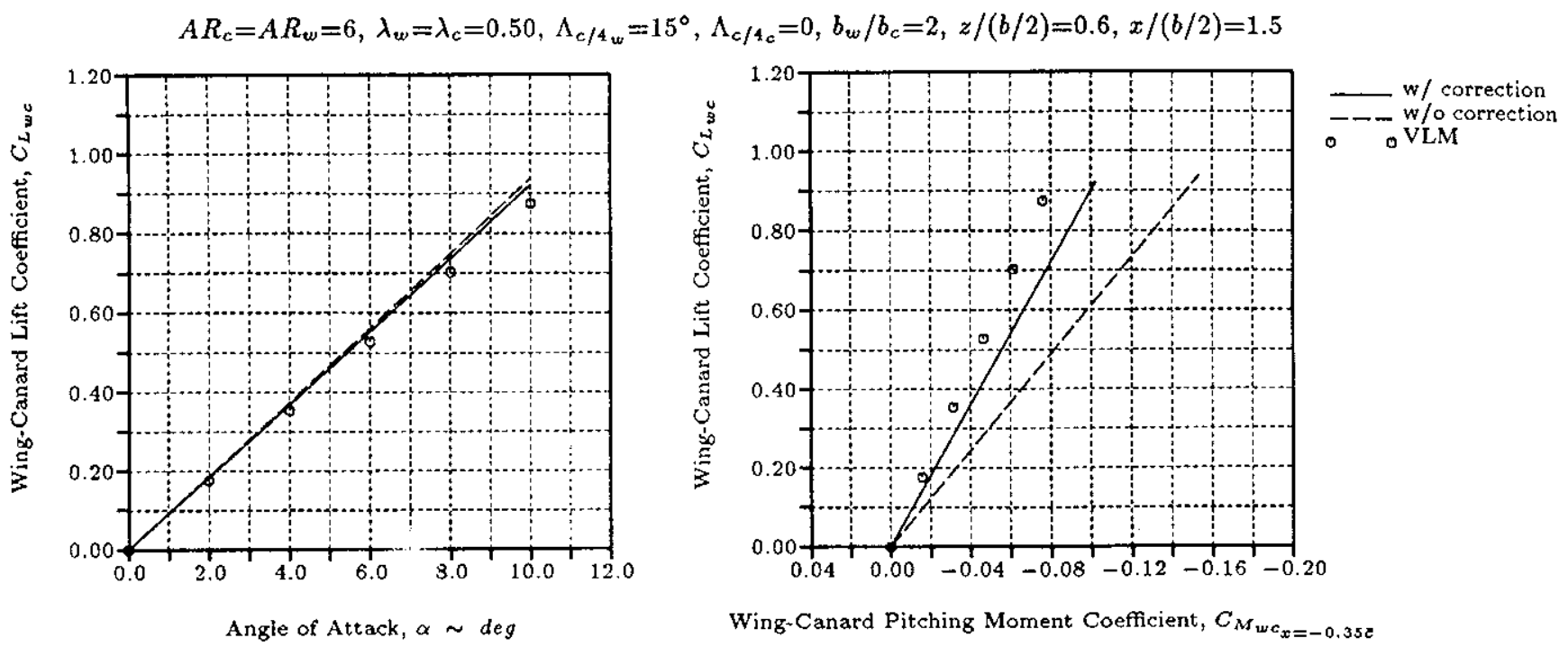

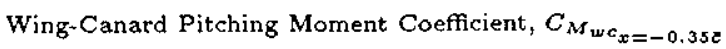

Figure 8: Predicted Lift and Pitching Moment Curves for a Wing-Canard Configuration With and Without Downwash Correction, Compared with Vortex Lattice Method Results, $\Lambda_{c / 4_{w}}=15^{\circ}$.

the exception of the wing lift curve slope:

$$
C_{L_{\alpha_{w}}}=.0772 \mathrm{deg}^{-1} \text {. }
$$

The result now is $C_{L_{\alpha_{w c}}}=.0921 \mathrm{deg}^{-1}$ with downwash corrections and $C_{L_{\alpha_{w c}}}=.0970 \mathrm{deg}^{-1}$ uncorrected. The wing-canard aerodynamic center locations are shown in Table 2 and the lift and pitching moment characteristics are shown in Figure 8.

It is seen that the lift is still fairly well predicted compared to the VLM results. The agreement between the aerodynamic center locations has improved somewhat. This is due to the moment generated on the wing, described above, due to the wing sweep. The net result is an estimated aerodynamic center location slightly too far forward.

\section{Conclusions and Recommenda- tions}

Design charts are presented which may be used to account for the effects of downwash on a wing due to a canard. The data are valid only for incompressible flow, but a wide range of planform parameters and geometric combinations are given. The data will not be accurate for cases where the canard wake impinges directly on the wing.

Greater accuracy can be obtained if the vortex core modeling includes viscous effects. The data would then be valid for cases where the canard wake passes more closely to the wing.

The present data are accurate enough to be used in conceptual and preliminary design studies. The prediction of the required canard size to satisfy lift and stability requirements can be better predicted by accounting for mutual interference effects.

\section{References}

[1] J. Roskam, Airplane Flight Dyanmics and Automatic Flight Controls. Roskam Aviation and Engineering Corporation, Ottowa, Kansas, 1979.

[2] A. Silverstein and S. Katzoff, "Design charts for predicting downwash angles and wake characteristics behind plain and flapped wings," NACA Technical Report 648, 1939.

[3] A. Silverstein et al, "Downwash and wake behind plain and flapped wings," NACA Technical Report $651,1939$.

[4] D. E. Hoak et al, USAF Stability and Control DATCOM. Global Engineering Documents, Clayton, MO, 1978.

[5] J. D. Phillips, "Downwash in the plane of symmetry of an elliptically loaded wing," NASA TP-2414, 1985.

[6] J. D. Phillips, "Approximate neutral point of a subsonic canard aircraft," NASA TM-86694, 1985.

[7] F. Ursell and G. N. Ward, "On some general theorems in the linearized theory of compressible flow," Quart. J. of Mechanics and Appl. Math., vol. 3, no. 3,1950 .

[8] J. Katz and A. Plotkin, Low-Speed Aerodynamics: From Wing Theory to Panel Methods. McGraw-Hill, 1991. 



Figure 9: Downwash Gradient in the Plane of Symmetry, $A R=6$. 


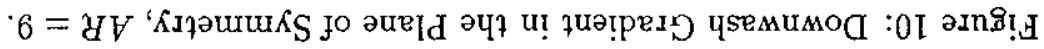

$(\tau / q) / x$

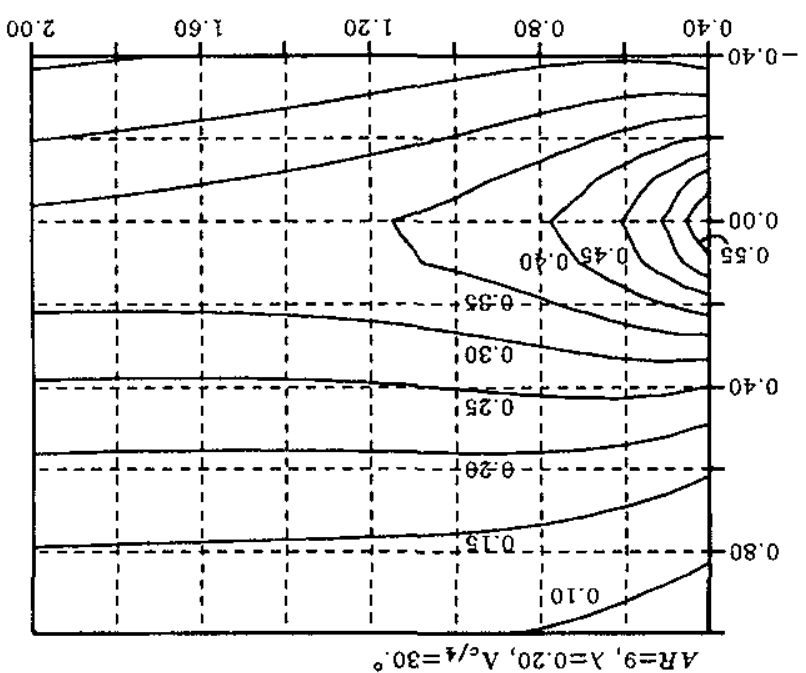

$(z / q) / x$

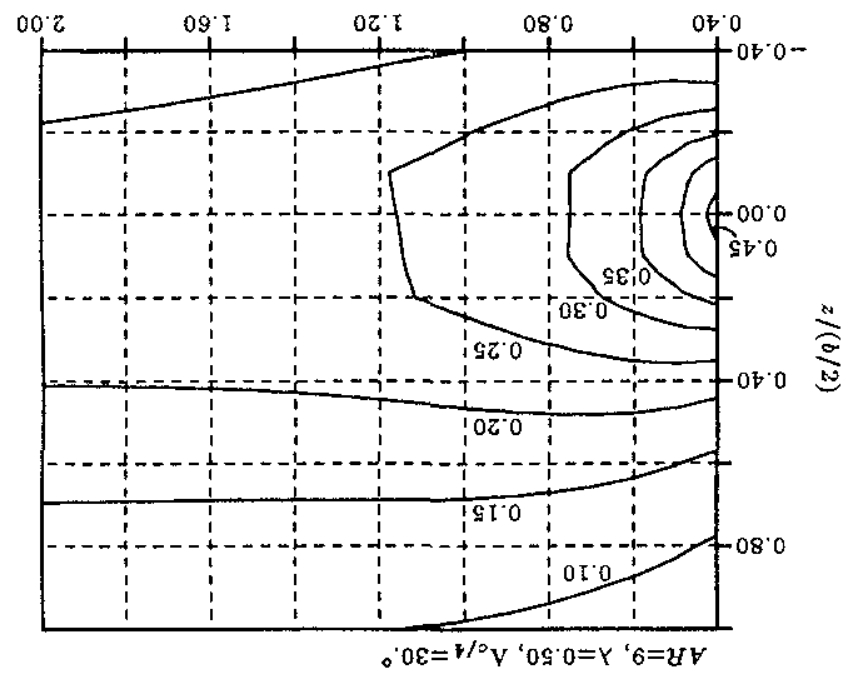

$(z / 9) / x$

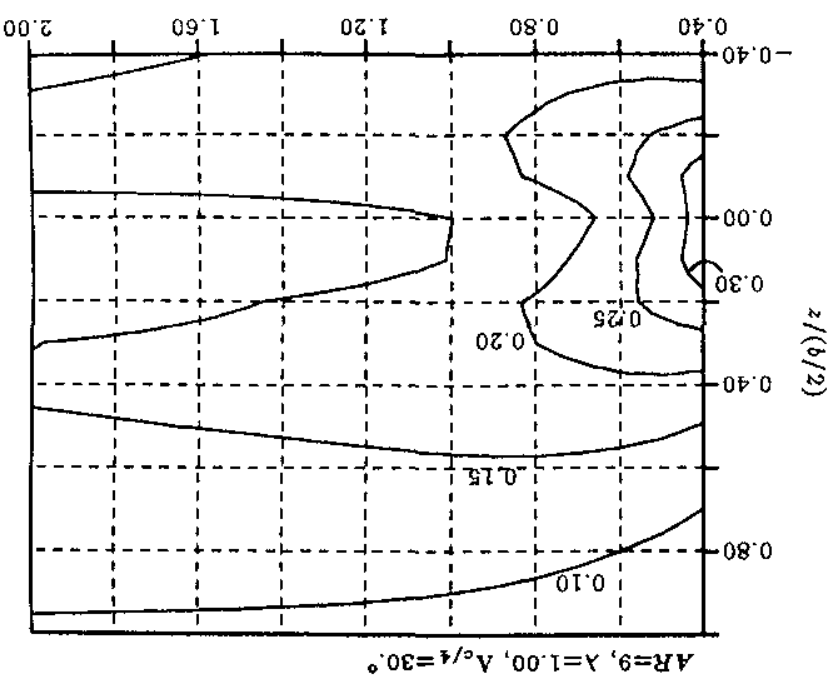

$(z / q) / x$

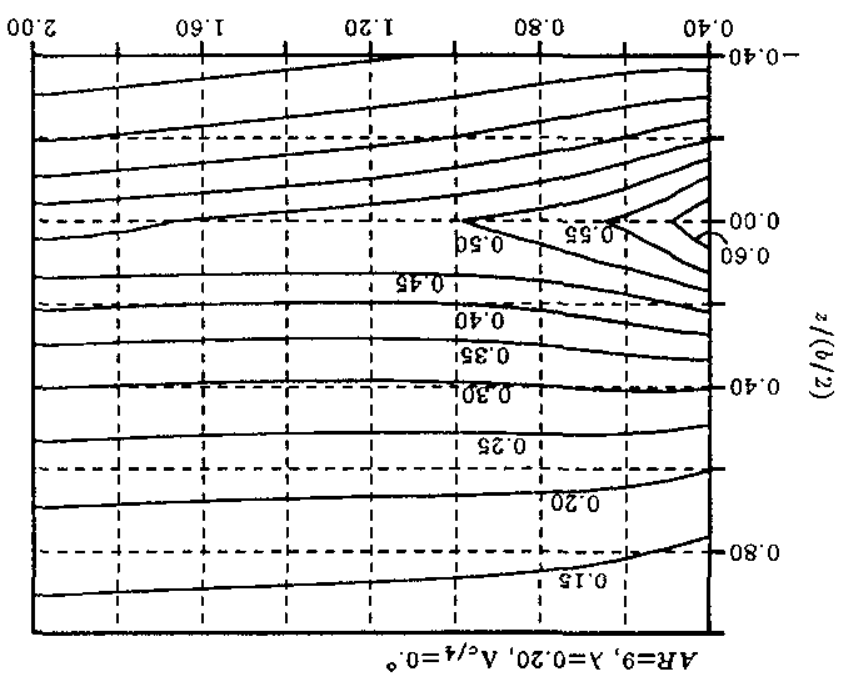

$(z / q) / x$

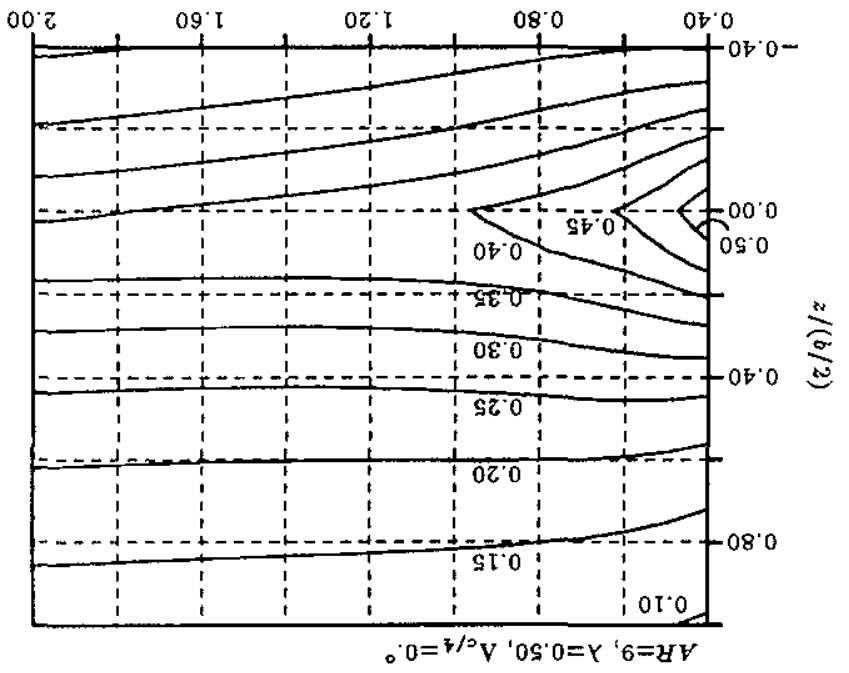

$(z / q) / x$

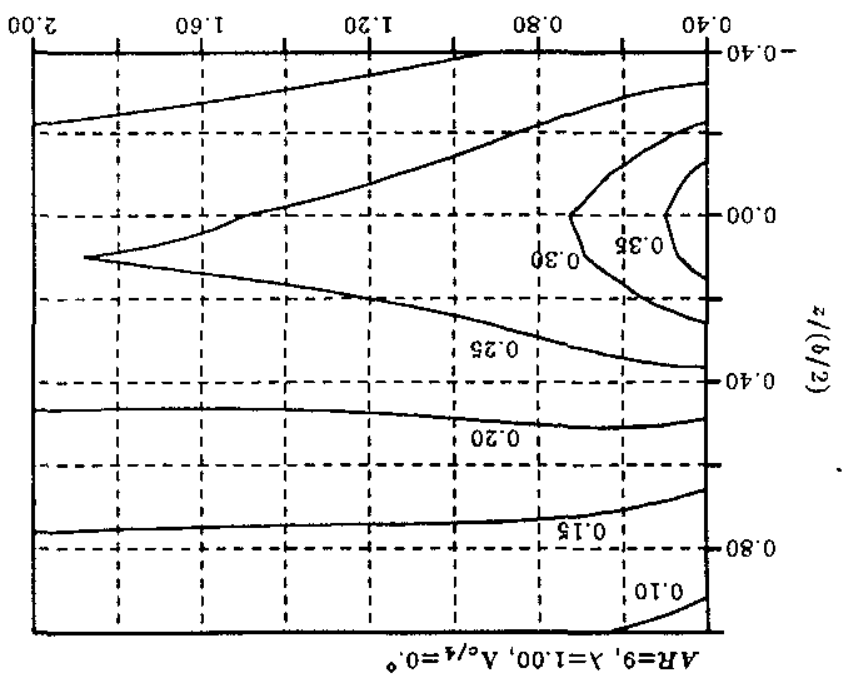



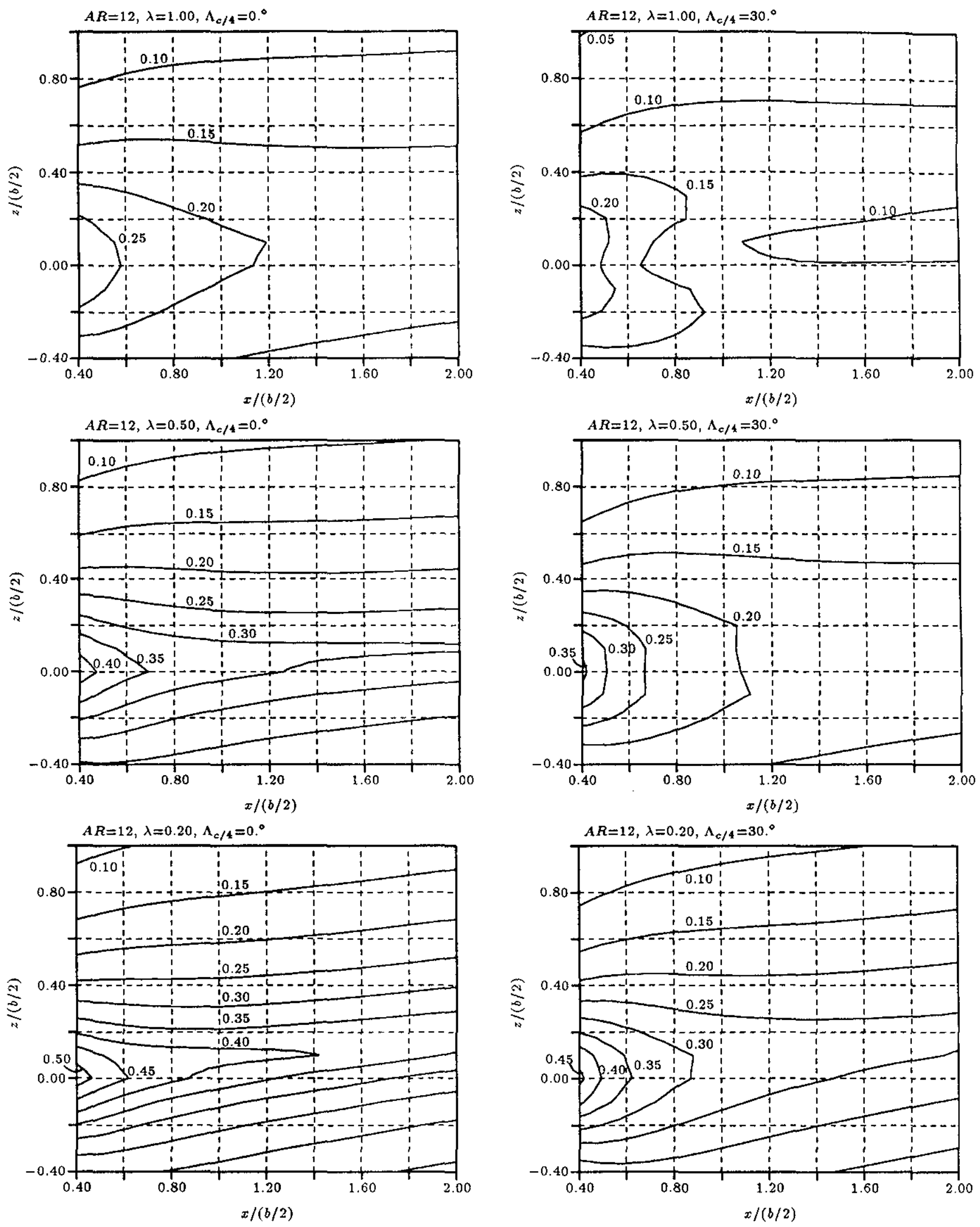

Figure 11: Downwash Gradient in the Plane of Symmetry, $A R=12$. 

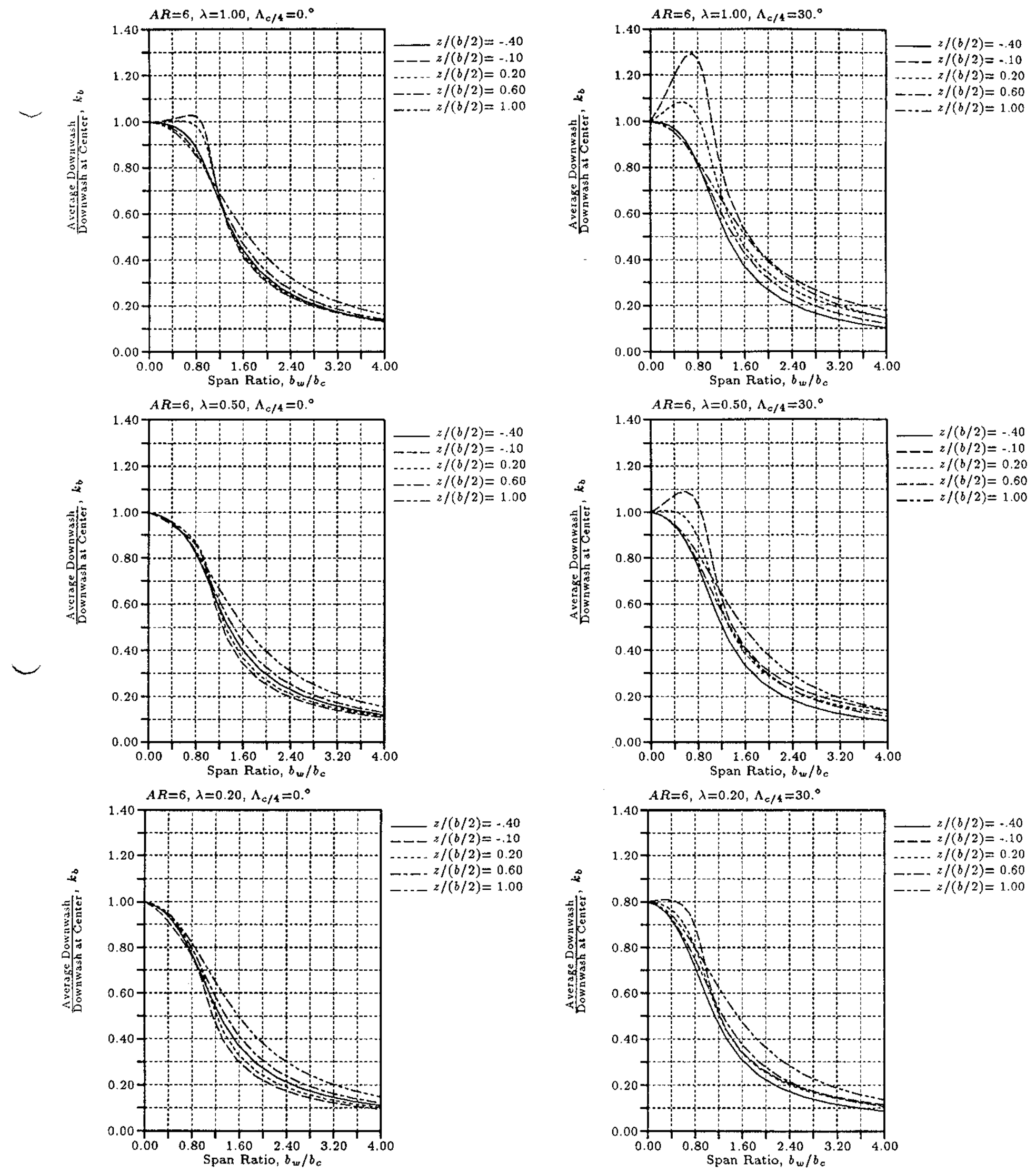

Figure 12: Correction Factor due to Spanwise Variation, $A R=6, x /(b / 2)=0.50$. 

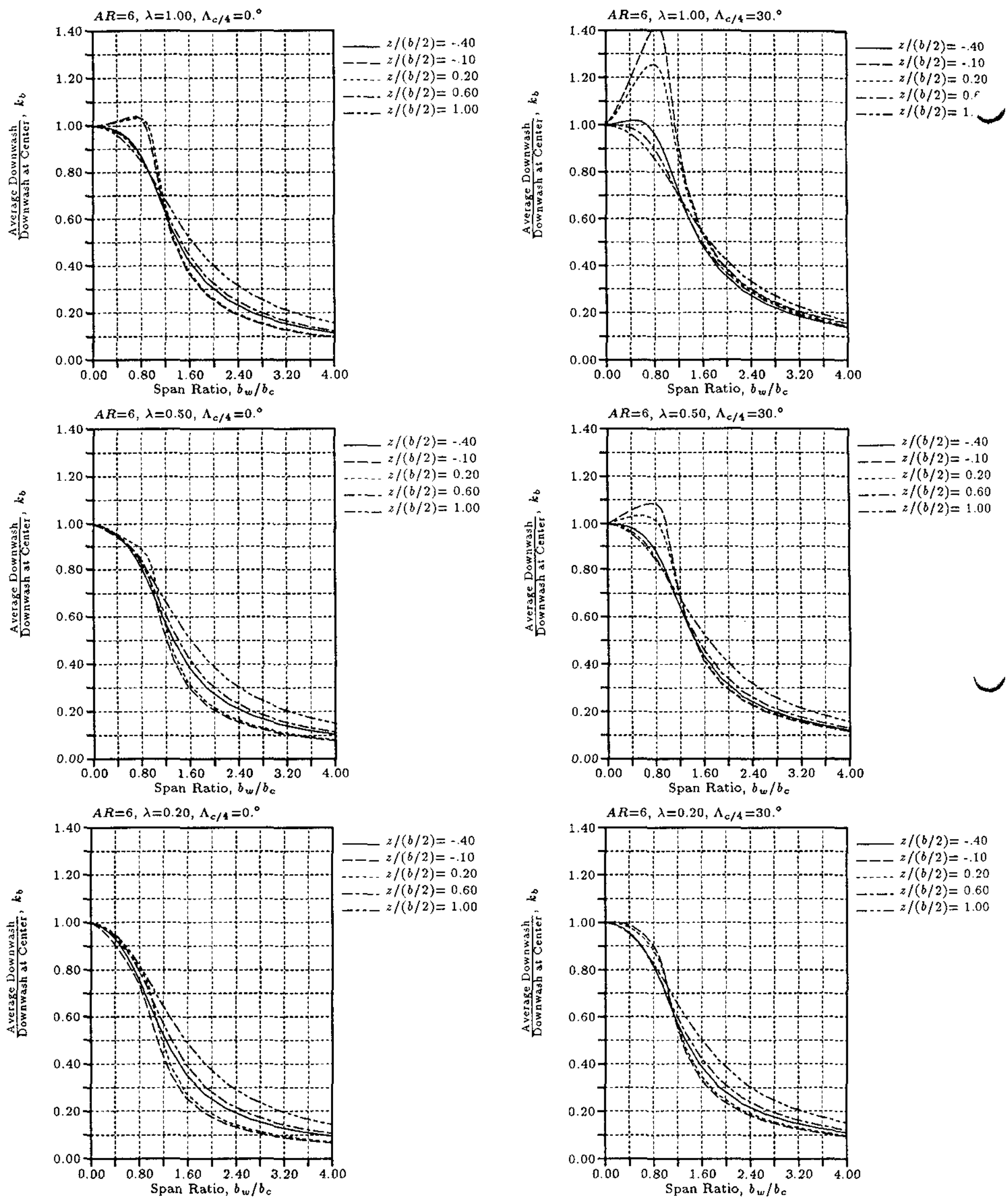

Figure 13: Correction Factor due to Spanwise Variation, $A R=6, x /(b / 2)=1.00$. 

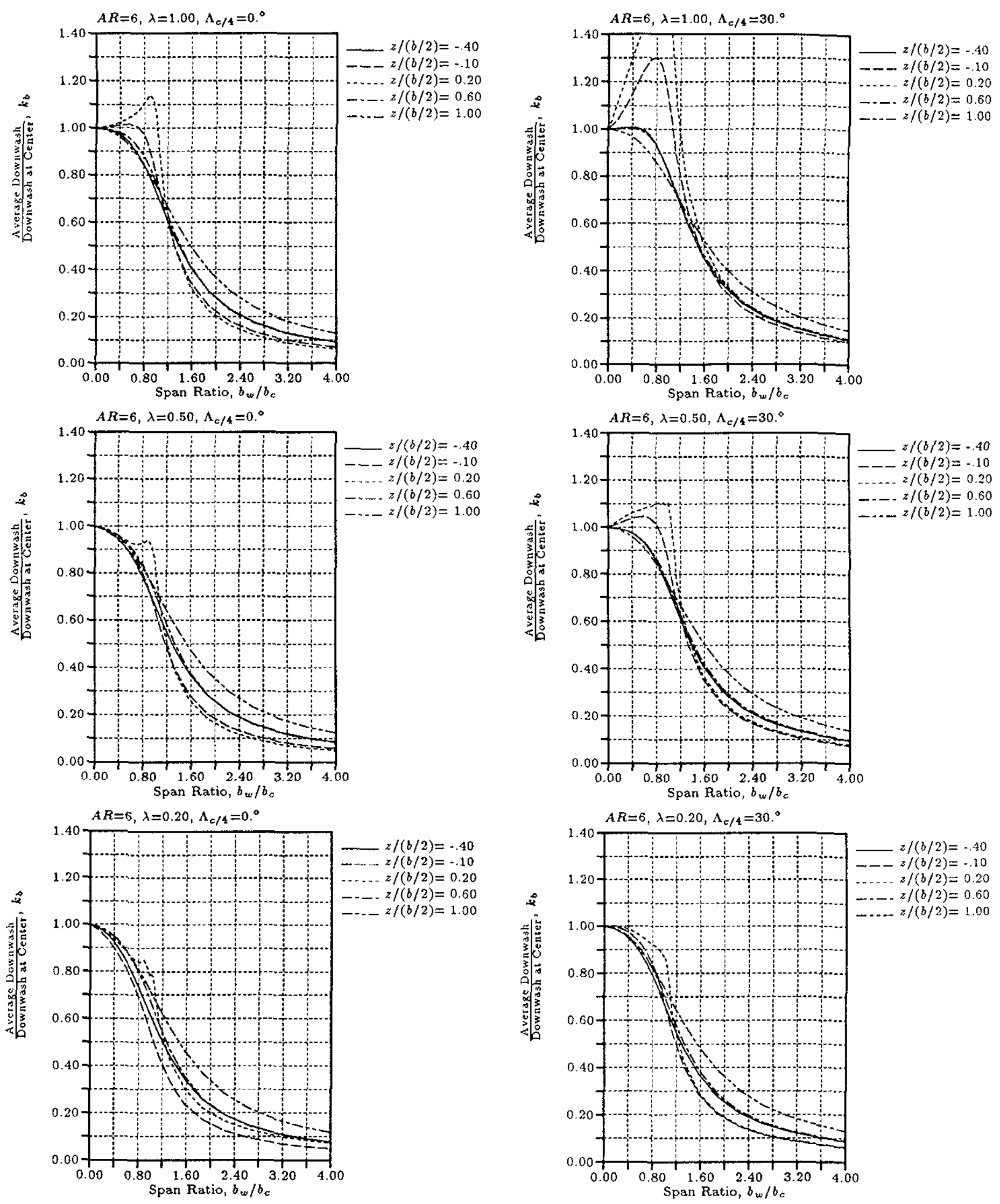

Figure 14: Correction Factor due to Spanwise Variation, $A R=6, x /(b / 2)=2.00$. 

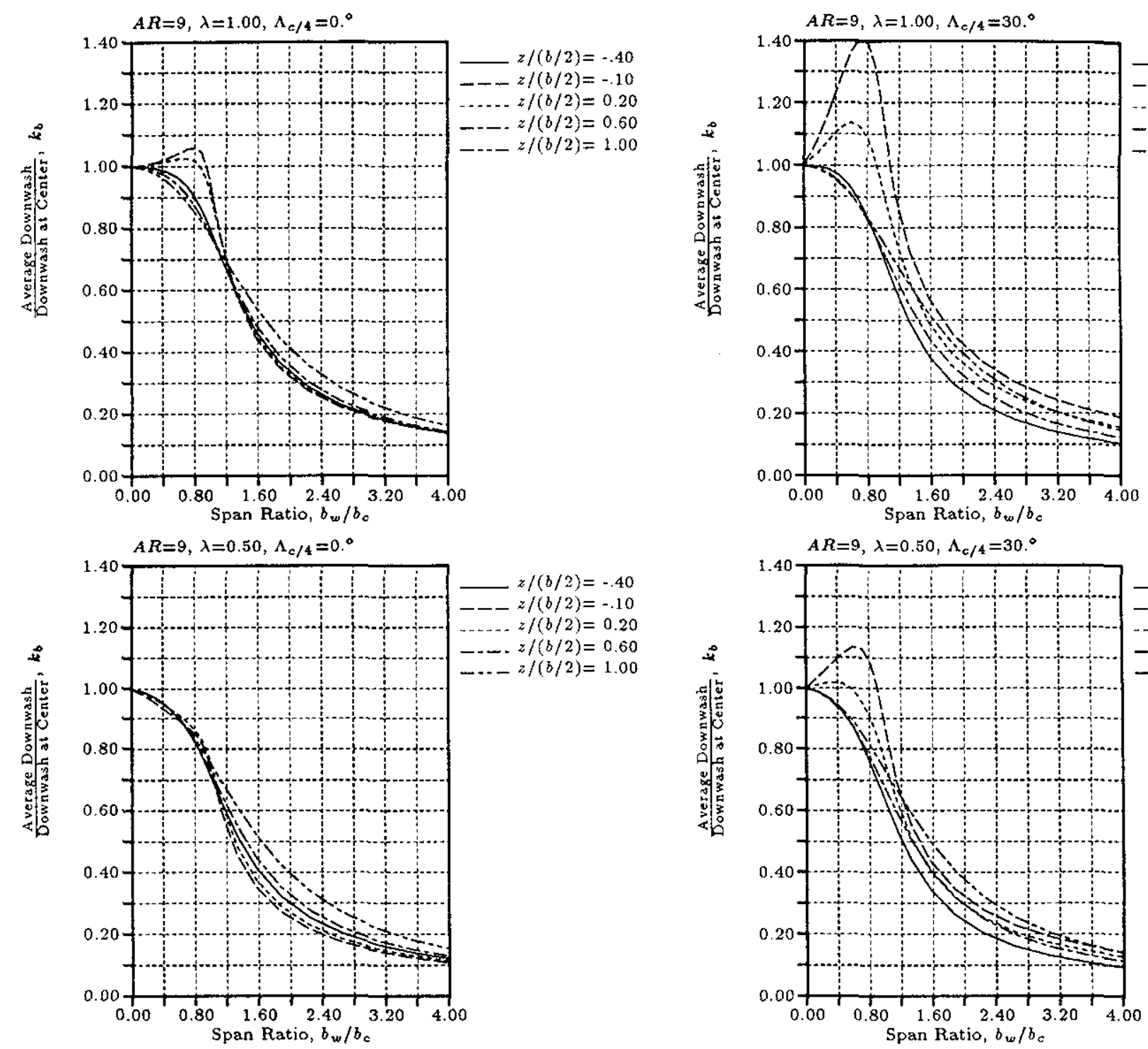

$z /(b / 2)=-.40$

$--z /(b / 2)=-.10$

$\ldots /(b / 2)=0.20$

$-\ldots z /(b / 2)=0.60$

$\cdots-z /(b / 2)=1.00$
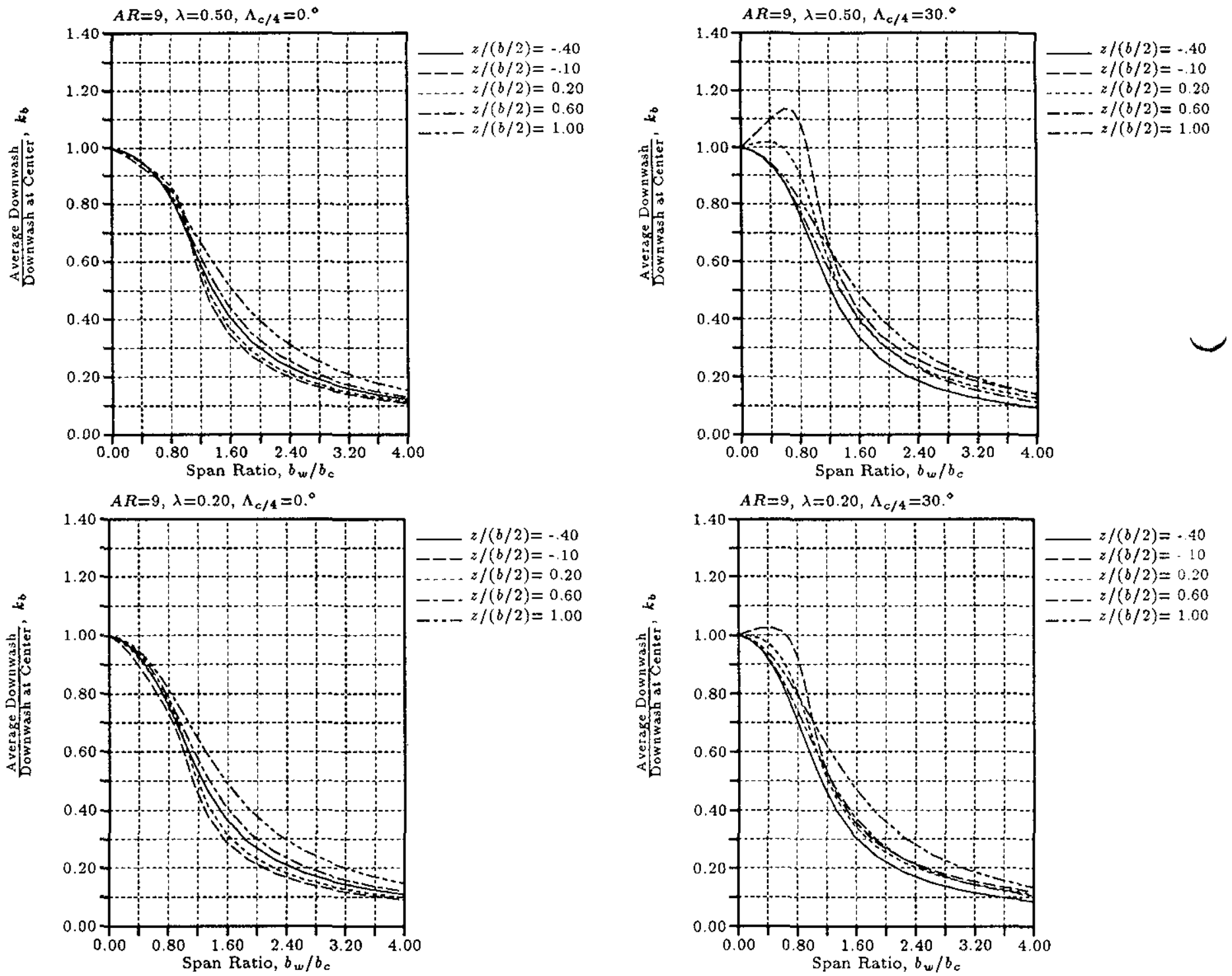

Figure 15: Correction Factor due to Spanwise Variation, $A R=9, x /(b / 2)=0.50$. 

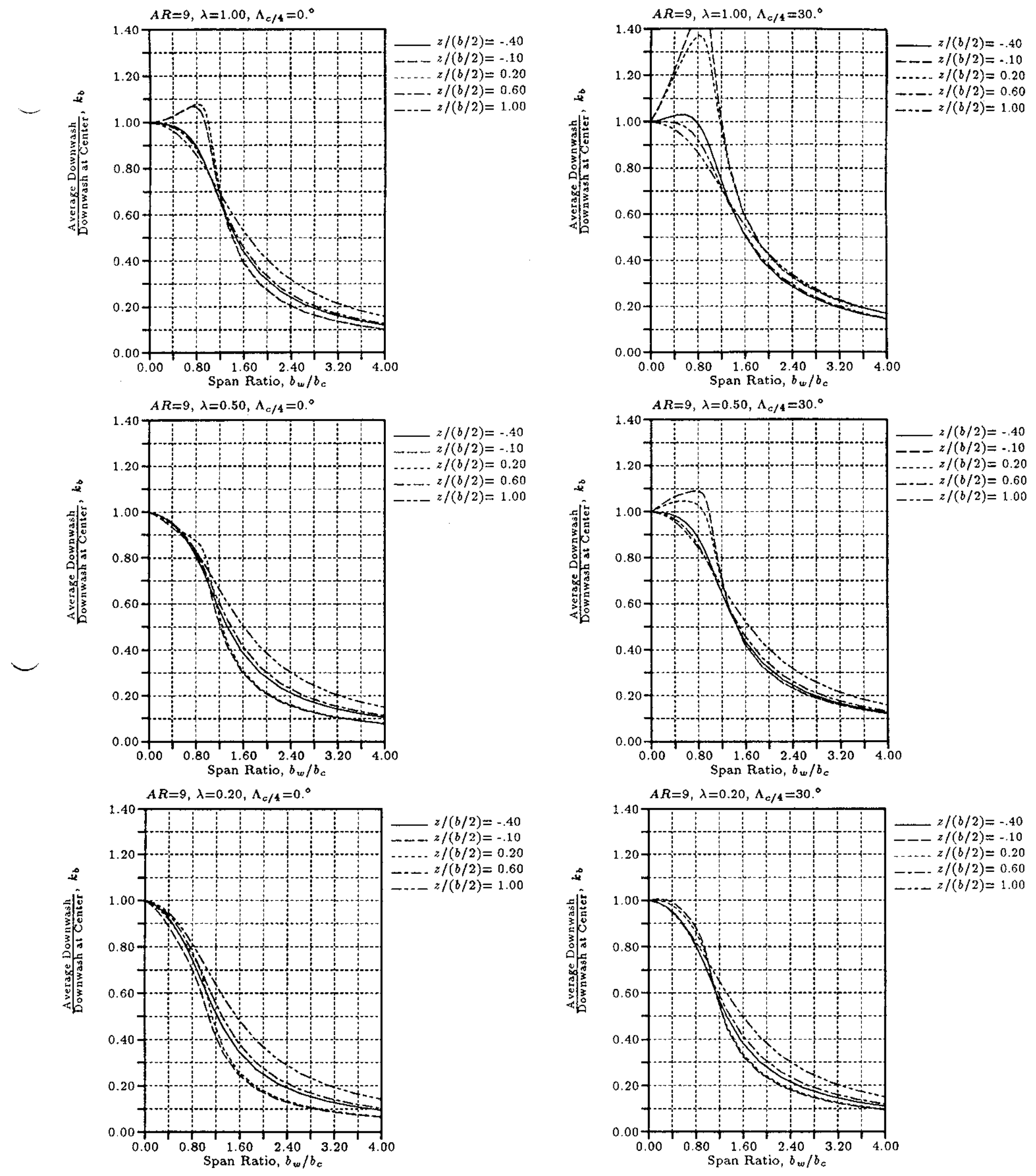

Figure 16: Correction Factor due to Spanwise Variation, $A R=9, x /(b / 2)=1.00$. 

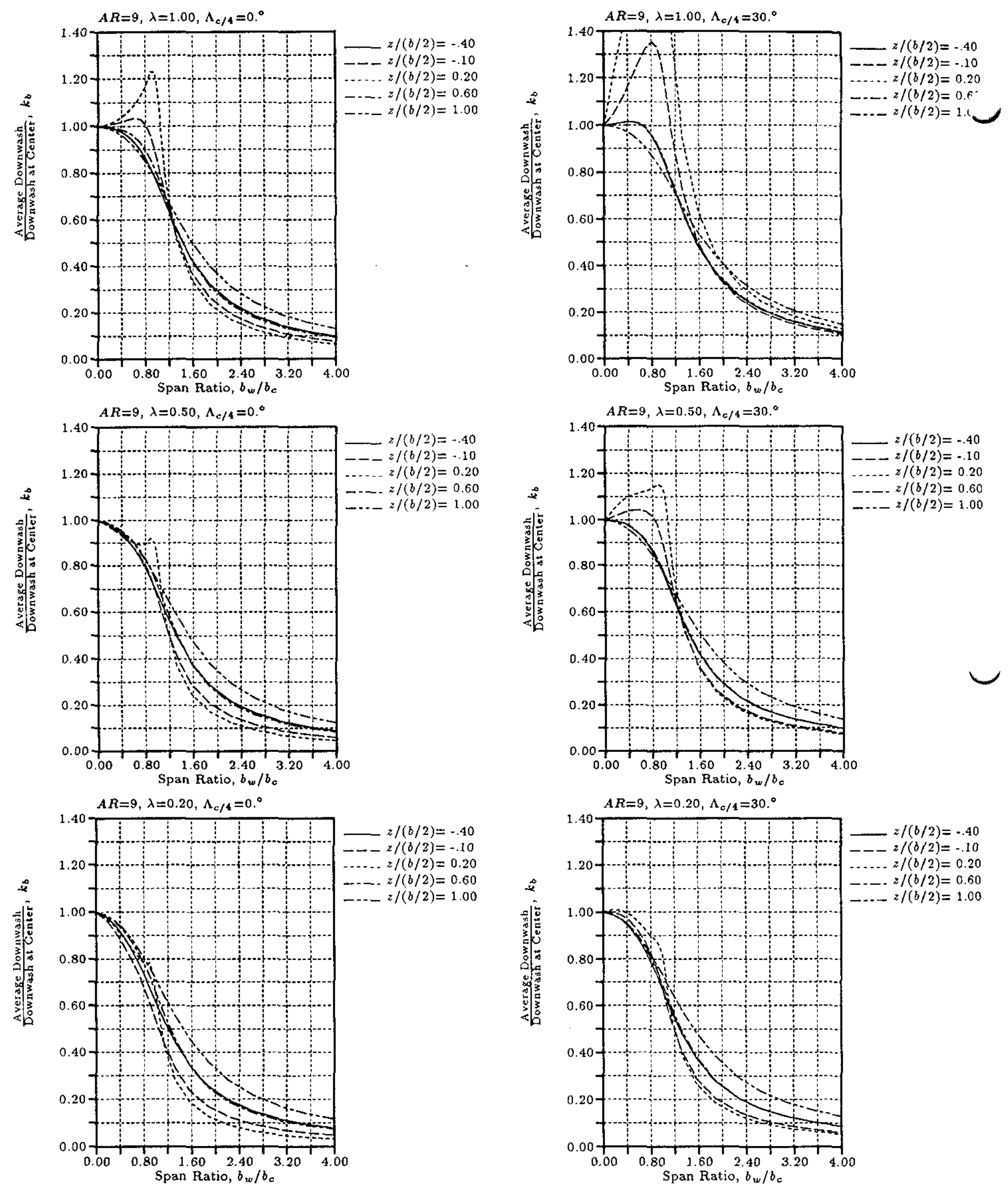

Figure 17: Correction Factor due to Spanwise Variation, $A R=9, x /(b / 2)=2.00$. 

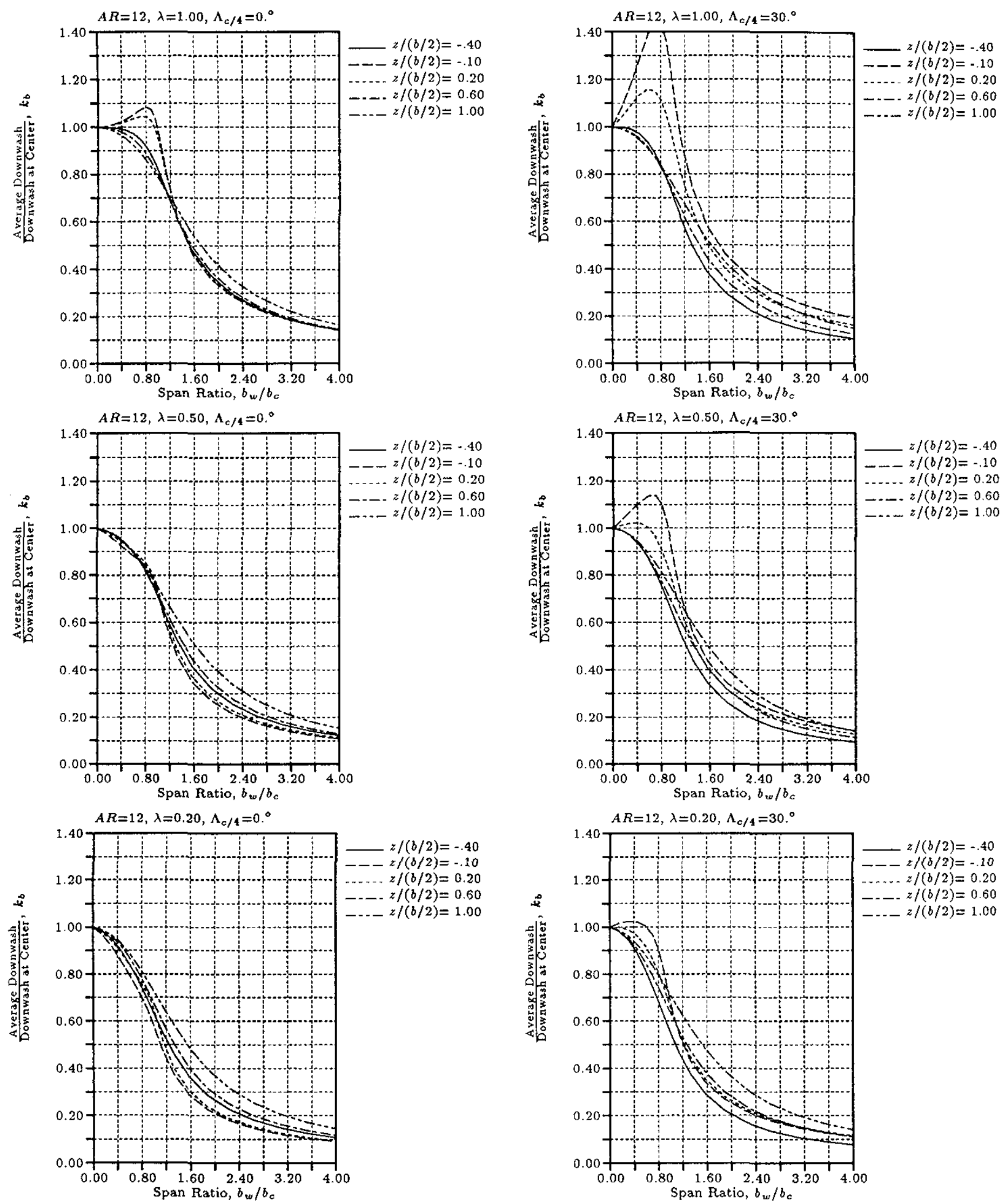

Figure 18: Correction Factor due to Spanwise Variation, $A R=12, x /(b / 2)=0.50$. 

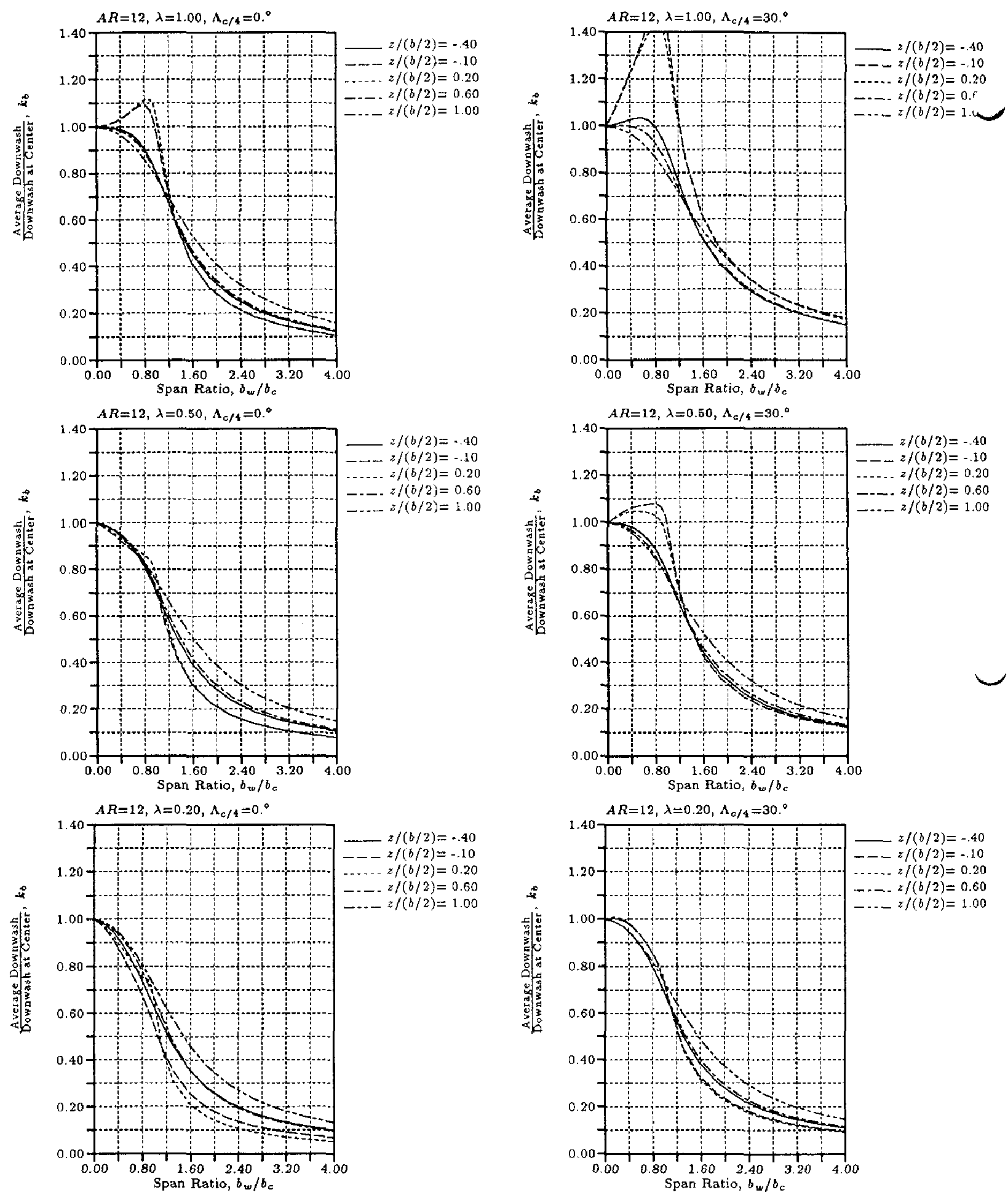

Figure 19: Correction Factor due to Spanwise Variation, $A R=12, x /(b / 2)=1.00$. 

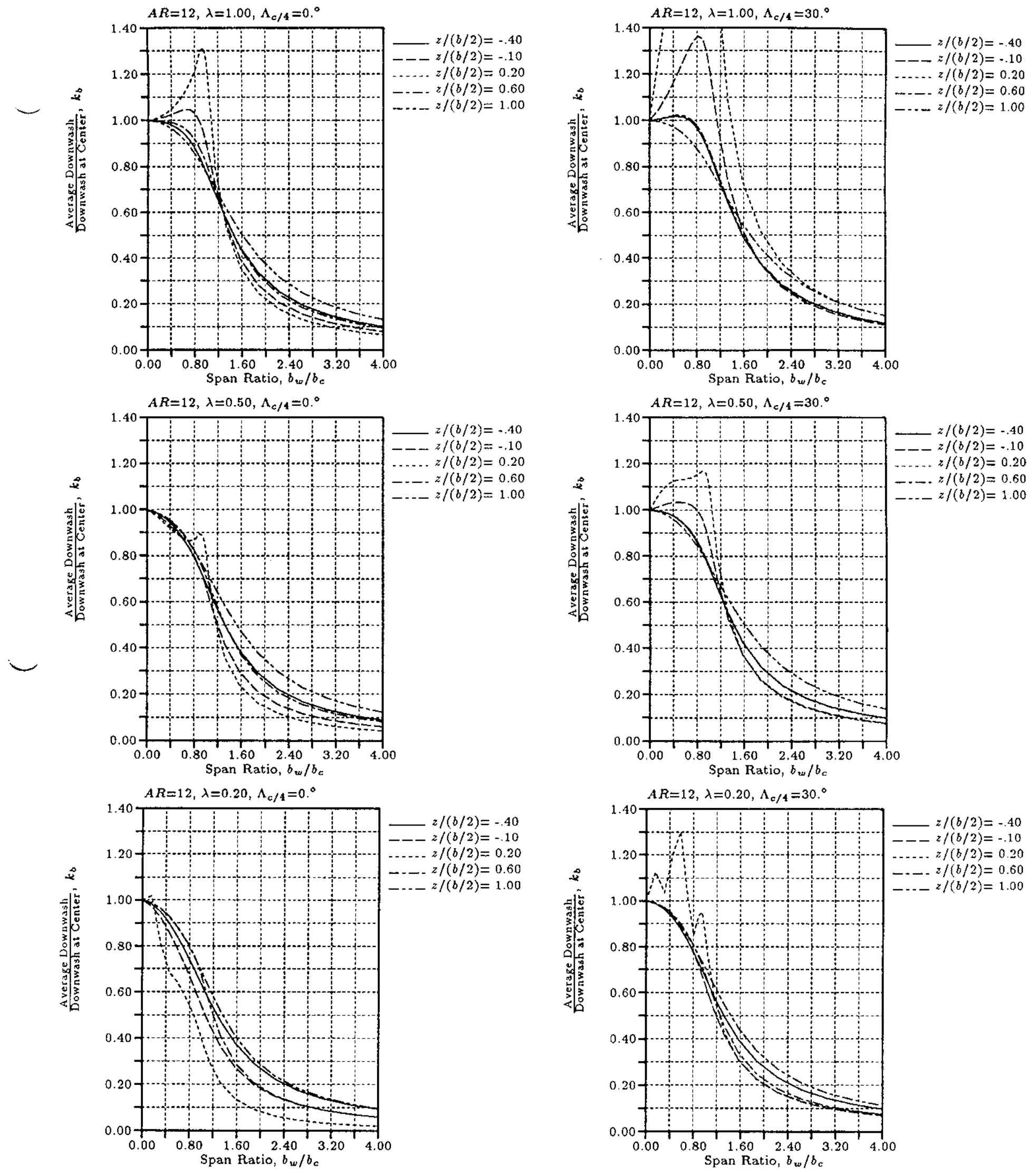

Figure 20: Correction Factor due to Spanwise Variation, $A R=12, x /(b / 2)=2.00$. 